\title{
Inequalities for the Mixed Radial Blaschke-Minkowski Homomorphisms and the Applications
}

\author{
Weidong Wang ${ }^{1,2}$ \\ ${ }^{1}$ Department of Mathematics, China Three Gorges University, Yichang, China \\ ${ }^{2}$ Three Gorges Mathematical Research Center, China Three Gorges University, Yichang, China
}

\section{Email address:}

wdwxh722@163.com

\section{To cite this article:}

Weidong Wang. CInequalities for the Mixed Radial Blaschke-Minkowski Homomorphisms and the Applications. Vol. 9, No. 1, 2020, pp. 14-19. doi: 10.11648/j.acm.20200901.12

Received: December 8, 2019; Accepted: December 19, 2019; Published: April 17, 2020

\begin{abstract}
The notion of intersection body is introduced by Lutwak in 1988, it is one of important research contents and led to the studies of Busemann-Petty problem in the Brunn-Minkowski theory. Based on the properties of the intersection bodies, Schuster introduced the notion of radial Blaschke-Minkowski homomorphisms and proved a lot of related inequalities. In this paper, by applying the dual mixed volume theory and analytic inequalities, we first give a lower bound of the dual quermassintegrals for the mixed radial Blaschke-Minkowski homomorphisms. As its an application, we get a reverse form of the well-known Busemann intersection inequality. Further, a Brunn-Minkowski type inequality of the $L_{p}$ radial Minkowski sum for the dual quermassintegrals of mixed radial Blaschke-Minkowski homomorphisms is established, and then the intersection body version of this Brunn-Minkowski type inequality is yielded. From this, we not only extend Schuster's related result but also obtain the Brunn-Minkowski type inequalities of $L_{p}$ harmonic radial sum and $L_{p}$ radial Blaschke sum, respectively.
\end{abstract}

Keywords: Dual Quermassintegral, Intersection Body, Radial Blaschke-Minkowski Homomorphism, Busemann Intersection Inequality, $L_{p}$ Radial Minkowski Sum

\section{Introduction}

The setting for this paper is Euclidean n-space $\mathbb{R}^{n}$. Let $S^{n-1}$ denote the unit sphere in $\mathbb{R}^{n}$ and $V(K)$ denote the $n$ dimensional volume of body $K$. For the centered unit $n$-ball $B$, write $V(B)=\omega_{n}$.

If $K$ is a compact star-shaped (about the origin) in $\mathbb{E}^{n}$, its radial function, $\rho_{K}=\rho(K, \cdot)$, is defined by [1]

$$
\rho(K, u)=\max \{\lambda \geq 0: \lambda u \in K\}
$$

for all $u \in S^{n-1}$. If $\rho_{K}$ is positive and continuous, $K$ will be called a star body (about the origin). Let $\mathcal{S}_{o}^{n}$ denote the set of all star bodies (about the origin) in $\mathbb{R}^{n}$.

The notion of intersection body was explicitly defined by Lutwak [2]. For $K \in \mathcal{S}_{o}^{n}$ and $n \geq 2$, the intersection body, $I K$, of $K$ as a star body whose radial function in direction $u \in S^{n-1}$ is equal to the $(n-1)$-dimensional volume of the section of $K$ by the hyperplane orthogonal to $u$, i.e.,

$$
\rho(I K, u)=v\left(K \cap u^{\perp}\right),
$$

where $v$ is the $n-1$-dimensional volume and $u^{\perp}$ is the $n-1$ dimensional subspace of $\mathbb{R}^{n}$ orthogonal to $u \in S^{n-1}$.

Intersection body is one of the central notions and led to the studies of Busemann-Petty problem in the Brunn-Minkowski theory $[1,3,4,2,5,6,7,8,9]$. Based on the properties of intersection bodies, Schuster [10] introduced the radial Blaschke-Minkowski homomorphisms as follows:

Definition 1.A A map $\Psi: \mathcal{S}_{o}^{n} \rightarrow \mathcal{S}_{o}^{n}$ is called a radial Blaschke-Minkowski homomorphism if it satisfies the following conditions:

(1) $\Psi$ is continuous.

(2) $\Psi K$ is radial Blaschke-Minkowski additive. i.e. $\Psi(K \widehat{+} L)=\Psi K \widetilde{+} \Psi L$ for all $K, L \in \mathcal{S}_{o}^{n}$. Here $K \widehat{+} L$ dentes the radial Blaschke sum of $K$ and $L$, and $\Psi K \widetilde{+} \Psi L$ denotes the radial Minkowski sum of $\Psi K$ and $\Psi L$.

(3) $\Psi$ intertwines rotations, i.e. $\Psi(\vartheta K)=\vartheta \Psi K$ for all $K \in \mathcal{S}_{o}^{n}$ and all $\vartheta \in S O(n)$.

According to above definition, Schuster [10] proved the following important result: 
Theorem 1.A There is a continuous operator

$$
\Psi: \underbrace{\mathcal{S}_{o}^{n} \times \cdots \times \mathcal{S}_{o}^{n}}_{n-1} \rightarrow \mathcal{S}_{o}^{n}
$$

symmetric in its arguments such that, for $L_{1}, \cdots, L_{m} \in \mathcal{S}_{o}^{n}$ and $\lambda_{1}, \cdots, \lambda_{m} \geq 0$,

$$
\Psi\left(\lambda_{1} L_{1} \tilde{+} \cdots \tilde{+} \lambda_{m} L_{m}\right)=\sum_{i_{1}, \cdots, i_{n-1}}^{\sim} \lambda_{i_{1}} \cdots \lambda_{i_{n-1}} \Psi\left(L_{i_{1}}, \cdots, L_{i_{n-1}}\right),
$$

where the sum is with respect to radial Minkowski addition.

Theorem 1.A generalizes the notion of radial Blaschke Minkowski homomorphism. We call

$$
\Psi: \underbrace{\mathcal{S}_{o}^{n} \times \cdots \times \mathcal{S}_{o}^{n}}_{n-1} \rightarrow \mathcal{S}_{o}^{n}
$$

the mixed radial Blaschke-Minkowski homomorphisms denoted by $\Psi\left(K_{1}, K_{2}, \cdots, K_{n-1}\right)$ for $K_{1}, K_{2}, \cdots, K_{n-1} \in$ $\mathcal{S}_{o}^{n}$.

For $K, L \in \mathcal{S}_{o}^{n}$, let $\Psi_{i}(K, L)=\Psi(\underbrace{K, \cdots, K}_{n-i-1}, \underbrace{L, \cdots, L}_{i})$ ( $i=0,1, \cdots, n-1$ ) denote the mixed radial BlaschkeMinkowski homomorphisms of $K$ and $L$. If $L=B$, we write $\Psi_{i} K=\Psi_{i}(K, B)$ and call $\Psi_{i} K$ the mixed radial BlaschkeMinkowski homomorphisms of $K$. Clearly, $\Psi_{0} K=\Psi K$.

Whereafter, Schuster [11] considered the Busemann-Petty problem for the radial Blaschke-Minkowski homomorphisms. In 2011, Wang, Liu and He [12] extended Schuster's radial Blaschke-Minkowski homomorphisms to $L_{p}$ analogies. In recent years, a lot of important conclusions for the radial Blaschke-Minkowski homomorphisms and their $L_{p}$ analogies were obtained, see e.g. $[13,14,15,16,17,12,18,19,20,21$, $22,23,24,25]$.

One purpose of this paper is to establish a lower bound for the dual quermassintegrals of the mixed radial BlaschkeMinkowski homomorphisms:

Theorem 1.1 If $K \in \mathcal{S}_{o}^{n}$, real $i<n-1$ and $j=$ $0,1, \cdots, n-1$, then

$$
\widetilde{W}_{i}\left(\Psi_{j} K\right) \geq \frac{r_{\Psi}^{n-i}}{\omega_{n}^{n-i-1}} \widetilde{W}_{j+1}(K)^{n-i}
$$

with equality if and only if $\Psi_{j} K$ is a ball. Here $r_{\Psi}$ denotes the radius of $\Psi B, \widetilde{W}_{i}(M)$ denotes the dual quermassintegrals of $M \in \mathcal{S}_{o}^{n}$.
Let $i=j=0$ in Theorem 1.1, and notice that $\Psi_{0} K=\Psi K$ and $\widetilde{W}_{0}(M)=V(M)$, we obtain that

Corollary 1.1 If $K \in \mathcal{S}_{o}^{n}$, then

$$
V(\Psi K) \geq \frac{r_{\Psi}^{n}}{\omega_{n}^{n-1}} \widetilde{W}_{1}(K)^{n}
$$

with equality if and only if $\Psi K$ is a ball. Here $r_{\Psi}$ denotes the radius of $\Psi B$.

Since the intersection operator $I$ is an example of a radial Blaschke-Minkowski homomorphism, thus, together with the radius of $I B$ is $\omega_{n-1}$, Corollary 1.1 provides the following a new inequality for the volume of intersection body.

Corollary 1.2 If $K \in \mathcal{S}_{o}^{n}$, then

$$
V(I K) \geq \frac{\omega_{n-1}^{n}}{\omega_{n}^{n-1}} \widetilde{W}_{1}(K)^{n},
$$

with equality if and only if $I K$ is a ball.

Remark 1.1 The fundamental volume inequality for intersection bodies is the well-known Busemann intersection inequality [26, 1]: If $K$ is a convex body (i.e. compact, convex subsets with non-empty interiors) containing the origin in its interiors in $\mathbb{R}^{n}$, then

$$
V(I K) \leq \frac{\omega_{n-1}^{n}}{\omega_{n}^{n-2}} V(K)^{n-1},
$$

with equality if and only if $K$ is a centered ellipsoid. Compare to inequality (1) and inequality (2), inequality (1) may be regards as a reverse form of Busemann intersection inequality.

In [10], Schuster proved a lot of inequalities for mixed radial Blaschke-Minkowski homomorphisms, especially, the following Brunn-Minkowski type inequality of radial sum of star bodies for mixed radial Blaschke-Minkowski homomorphisms was given.

Theorem 1.B If $K, L \in \mathcal{S}_{o}^{n}, i=0,1, \cdots, n-2$ and $j=0,1, \cdots, n-2$, then

$$
\widetilde{W}_{i}\left(\Psi_{j}(K \widetilde{+} L)\right)^{\frac{1}{(n-i)(n-j-1)}} \leq \widetilde{W}_{i}\left(\Psi_{j} K\right)^{\frac{1}{(n-i)(n-j-1)}}+\widetilde{W}_{i}\left(\Psi_{j} L\right)^{\frac{1}{(n-i)(n-j-1)}},
$$

with equality if and only if $K$ and $L$ are dilates. Here, $K \widetilde{+} L$ denotes the radial sum of $K$ and $L$.

Our next result generalizes the above Brunn-Minkowski type inequality to $L_{p}$ radial Minkowski sum of star bodies.

Theorem 1.2 Let $K, L \in \mathcal{S}_{o}^{n}$, real $p \neq 0, i=0,1, \cdots, n-2$ and $j$ is an integer. If $p<0$ and $0 \leq j<n-1$, then

$$
\widetilde{W}_{i}\left(\Psi_{j}\left(K \widetilde{+}_{p} L\right)\right)^{\frac{p}{(n-i)(n-j-1)}} \geq \widetilde{W}_{i}\left(\Psi_{j} K\right)^{\frac{p}{(n-i)(n-j-1)}}+\widetilde{W}_{i}\left(\Psi_{j} L\right)^{\frac{p}{(n-i)(n-j-1)}} ;
$$


if $0<p<n-1$ and $0 \leq j<n-p-1$, then

$$
\widetilde{W}_{i}\left(\Psi_{j}\left(K \widetilde{+}_{p} L\right)\right)^{\frac{p}{(n-i)(n-j-1)}} \leq \widetilde{W}_{i}\left(\Psi_{j} K\right)^{\frac{p}{(n-i)(n-j-1)}}+\widetilde{W}_{i}\left(\Psi_{j} L\right)^{\frac{p}{(n-i)(n-j-1)}} .
$$

In each inequality, equality holds if and only if $K$ and $L$ are dilates. Here, $K \widetilde{+}_{p} L$ denotes the $L_{p}$-radial sum of $K$ and $L$.

Remark 1.2 If $p=1$ in Theorem 1.2, then inequality (4) just is inequality (3). Let $i=j=0$ in Theorem 1.2, and notice intersection operator $I$ is a special case of radial Blaschke-Minkowski homomorphisms, we have

Corollary 1.3 Let $K, L \in \mathcal{S}_{o}^{n}$, real $p \neq 0$. If $p<0$, then

$$
V\left(I\left(K \widetilde{+}_{p} L\right)\right)^{\frac{p}{n(n-1)}} \geq V(I K)^{\frac{p}{n(n-1)}}+V(I L)^{\frac{p}{n(n-1)}}
$$

if $0<p<n-1$, then

$$
V\left(I\left(K \widetilde{+}_{p} L\right)\right)^{\frac{p}{n(n-1)}} \leq V(I K)^{\frac{p}{n(n-1)}}+V(I L)^{\frac{p}{n(n-1)}} .
$$

In each inequality, equality holds if and only if $K$ and $L$ are dilates.

Remark 1.3 Let $p=-q(q \geq 1)$ or $p=n-q(1<q<n)$ in Theorem 1.2, respectively, then inequality (4) orderly gives the Brunn-Minkowski type inequalities for the $L_{q}$ harmonic radial sum and the $L_{q}$ radial Blaschke sum as follows:

Corollary 1.4 If $K, L \in \mathcal{S}_{o}^{n}$, real $q \geq 1, i=0,1, \cdots, n-2$ and $j=0,1, \cdots, n-2$, then

$$
\widetilde{W}_{i}\left(\Psi_{j}\left(K \widetilde{+}_{-q} L\right)\right)^{-\frac{q}{(n-i)(n-j-1)}} \geq \widetilde{W}_{i}\left(\Psi_{j} K\right)^{-\frac{q}{(n-i)(n-j-1)}}+\widetilde{W}_{i}\left(\Psi_{j} L\right)^{-\frac{q}{(n-i)(n-j-1)}},
$$

with inequality if and only if $K$ and $L$ are dilates. Here, $K \widetilde{+}_{-q} L$ denotes the $L_{q}$ harmonic radial sum of $K$ and $L$.

Corollary 1.5 If $K, L \in \mathcal{S}_{o}^{n}$, real $1<q<n, i=0,1, \cdots, n-2$ and integer $j$ satisfies $0 \leq j<q-1$, then

$$
\widetilde{W}_{i}\left(\Psi_{j}\left(K \widehat{+}_{q} L\right)\right)^{\frac{n-q}{(n-i)(n-j-1)}} \leq \widetilde{W}_{i}\left(\Psi_{j} K\right)^{\frac{n-q}{(n-i)(n-j-1)}}+\widetilde{W}_{i}\left(\Psi_{j} L\right)^{\frac{n-q}{(n-i)(n-j-1)}}
$$

with inequality if and only if $K$ and $L$ are dilates. Here, $K \widehat{+}_{q} L$ denotes the $L_{q}$-radial Blaschke sum of $K$ and $L$.

Corollary 1.4 and Corollary 1.5 were established by Wei, Wang and Lu [19].

\section{Notations and Background Materials}

\section{1. $L_{p}$ Radial Minkowski Combinations}

The $L_{p}$ radial Minkowski combination of star bodies was introduced as follows [27, 7, 28]: For $K, L \in \mathcal{S}_{o}^{n}$, real $p \neq 0$ and $\lambda, \mu \geq 0$ (nor both 0 ), the $L_{p}$ radial Minkowski combination, $\lambda \cdot K \widetilde{+}_{p} \mu \cdot L$, of $K$ and $L$ is defined by

$$
\rho\left(\lambda \cdot K \widetilde{+}_{p} \mu \cdot L, \cdot\right)^{p}=\lambda \rho(K, \cdot)^{p}+\mu \rho(L, \cdot)^{p} .
$$

Here " $\widetilde{+}_{p}$ " denotes the $L_{p}$ radial Minkowski sum, $\lambda \cdot K$ denotes the $L_{p}$ radial Minkowski scalar multiplication and $\lambda \cdot K=\lambda^{1 / p} K$. The case $p=1$ yields the radial Minkowski combination $\lambda \cdot K \widetilde{+} \mu \cdot L$.

Let $p=-q(q \geq 1)$ in (6), then $\lambda \cdot K \widetilde{+}_{-q} \mu \cdot L$ is called the $L_{q}$ harmonic radial combination of star bodies $K$ and $L[1,7]$.

In 2015, Wang and Wang [29] defined the $L_{p}$ radial Blaschke combinations of star bodies as follows: For $K, L \in$ $\mathcal{S}_{o}^{n}, n>p>0$ and $\lambda, \mu \geq 0$ (not both 0$)$, the $L_{p}$ radial Blaschke combination, $\lambda \circ K \widehat{+}_{p} \mu \circ L \in \mathcal{S}_{o}^{n}$, of $K$ and $L$ is defined by

$$
\rho\left(\lambda \circ K \widehat{+}_{p} \mu \circ L, \cdot\right)^{n-p}=\lambda \rho(K, \cdot)^{n-p}+\mu \rho(L, \cdot)^{n-p} .
$$

Here " $\widehat{+}_{p}$ " denotes the $L_{p}$ radial Blaschke sum, $\lambda \circ K$ denotes the $L_{p}$ radial Blaschke scalar multiplication and $\lambda \circ$ $K=\lambda^{1 /(n-p)} K$. Clearly, (6) and (7) show that $\lambda \circ K \widehat{+}_{p} \mu \circ$ $L=\lambda \cdot K \widetilde{+}_{n-p} \mu \cdot L$.

\subsection{Dual Mixed Volumes}

In 1975, Lutwak [30] introduced the dual mixed volumes as follows: For $K_{1}, \cdots, K_{n} \in \mathcal{S}_{o}^{n}$, the dual mixed volume $\widetilde{V}\left(K_{1}, \cdots, K_{n}\right)$ is defined by

$$
\widetilde{V}\left(K_{1}, K_{2}, \cdots, K_{n}\right)=\frac{1}{n} \int_{S^{n-1}} \rho_{K_{1}}(u) \rho_{K_{2}}(u) \cdots \rho_{K_{n}}(u) d u .
$$

For $K, L \in \mathcal{S}_{o}^{n}$ and $i=0,1,2, \cdots, n$, we write $\widetilde{V}_{i}(K, L)$ to denote the dual mixed volume $\widetilde{V}_{i}(\underbrace{K, \cdots, K}_{n-i}, \underbrace{L, \cdots, L}_{i})$. Similarly, we use $\widetilde{W}_{i}(K, L)=\widetilde{V}_{i}(\underbrace{K, \cdots, K}_{n-i-1}, \underbrace{B, \cdots, B}_{i}, L)$ to denote the dual mixed quermassintegrals of $K$ and $L$. If let $i$ be real, then an extension of the dual mixed volume $\widetilde{V}_{i}(K, L)$ is that for $K, L \in \mathcal{S}_{o}^{n}$ and $i \in \mathbb{R}$ :

$$
\widetilde{V}_{i}(K, L)=\frac{1}{n} \int_{S^{n-1}} \rho(K, u)^{n-i} \rho(L, u)^{i} d u .
$$

In the same way, for $K, L \in \mathcal{S}_{o}^{n}$ and $i \in \mathbb{R}$, we define the dual mixed quermassintegrals of $K$ and $L$ by

$$
\widetilde{W}_{i}(K, L)=\frac{1}{n} \int_{S^{n-1}} \rho(K, u)^{n-i-1} \rho(L, u) d u .
$$

Let $L=B$ in (8) (or $L=K$ in (9)), then the dual 
quermassintegrals, $\widetilde{W}_{i}(K)$, of $K \in \mathcal{S}_{o}^{n}$ is given by

$$
\begin{aligned}
\widetilde{W}_{i}(K) & =\widetilde{V}_{i}(K, B)=\widetilde{W}_{i}(K, K) \\
& =\frac{1}{n} \int_{S^{n-1}} \rho_{K}^{n-i}(u) d u .
\end{aligned}
$$

Further let $i=0$ in (10), then we have the following polar coordinate formula for the volume of a body $K$ :

$$
V(K)=\widetilde{W}_{0}(K)=\frac{1}{n} \int_{S^{n-1}} \rho_{K}^{n}(u) d u .
$$

For the above dual mixed quermassintegrals, corresponding the Minkowski inequality is stated that [10]: If $K, L \in \mathcal{S}_{o}^{n}$ and real $i$ satisfies $0 \leq i<n-1$, then

$$
\widetilde{W}_{i}(K, L) \leq \widetilde{W}_{i}(K)^{\frac{n-i-1}{n-i}} \widetilde{W}_{i}(L)^{\frac{1}{n-i}},
$$

with equality if and only if $K$ and $L$ are dilates.

\section{Proofs of the Main Results}

In this section we will prove Theorems 1.1 and 1.2. To see this, the following lemmas is required.

Lemma 3.1 [30] If $K, L \in \mathcal{S}_{0}^{n}$, and reals $i, j, k$ satisfy $i<j<k$, then

$$
\widetilde{V}_{j}(K, L)^{k-i} \leq \widetilde{V}_{i}(K, L)^{k-j} \widetilde{V}_{k}(K, L)^{j-i},
$$

with equality if and only if $K$ and $L$ are dilates.
Lemma 3.2 [10] If $L \in \mathcal{S}_{o}^{n}, i=0,1, \cdots, n-2$, then

$$
\widetilde{W}_{n-1}\left(\Psi_{i} L\right)=r_{\Psi} \widetilde{W}_{i+1}(L) .
$$

Proof of Theorem 1.1 In (12), let $L=B, j=n-1$ and $k=n$, and notice that $\widetilde{V}_{n}(K, B)=\omega_{n}$ by (8), we have for $i<n-1$,

$$
\widetilde{W}_{n-1}(K)^{n-i} \leq \omega_{n}^{n-i-1} \widetilde{W}_{i}(K),
$$

with equality if and only if $K$ is a ball centrad at the origin.

Taking $\Psi_{j} K$ for $K$ in (14), we get for $j=0,1, \cdots, n-1$,

$$
\widetilde{W}_{n-1}\left(\Psi_{j} K\right)^{n-i} \leq \omega_{n}^{n-i-1} \widetilde{W}_{i}\left(\Psi_{j} K\right) .
$$

This and (13) yield

$$
r_{\Psi}^{n-i} \widetilde{W}_{j+1}(K)^{n-i} \leq \omega_{n}^{n-i-1} \widetilde{W}_{i}\left(\Psi_{j} K\right),
$$

with equality if and only if $\psi_{j} K$ is a ball centrad at the origin.

Thus, we have

$$
\widetilde{W}_{i}\left(\Psi_{j} K\right) \geq \frac{r_{\Psi}^{n-i} \widetilde{W}_{j+1}(K)^{n-i}}{\omega_{n}^{n-i-1}}
$$

with equality if and only if $\psi_{j} K$ is a ball centrad at the origin. This gives Theorem 1.1.

Now we give the proof of Theorem 1.2. Here, we prove a lemma as follows:

Lemma 3.3 Let $K, L \in \mathcal{S}_{o}^{n}$, real $p \neq 0$, and $j$ is an integer. If $p<0$ and $0 \leq j<n-1$, then

$$
\widetilde{W}_{j}\left(K \widetilde{+}_{p} L, Q\right)^{\frac{p}{n-j-1}} \geq \widetilde{W}_{i}(K, Q)^{\frac{p}{n-j-1}}+\widetilde{W}_{i}(L, Q)^{\frac{p}{n-j-1}}
$$

if $0<p<n-1$ and $0 \leq j<n-p-1$, then

$$
\widetilde{W}_{j}\left(K \widetilde{+}_{p} L, Q\right)^{\frac{p}{n-j-1}} \leq \widetilde{W}_{i}(K, Q)^{\frac{p}{n-j-1}}+\widetilde{W}_{i}(L, Q)^{\frac{p}{n-j-1}} .
$$

In each inequality, equality holds if and only if $K$ and $L$ are dilates.

Proof. From (6), (9) and the Minkowski integral inequality, if $p<0$ and $0 \leq j<n-1$, then $\frac{n-j-1}{p}<0$. Thus, for any $Q \in \mathcal{S}_{o}^{n}$,

$$
\begin{aligned}
\widetilde{W}_{j}\left(K \widetilde{+}_{p} L, Q\right)^{\frac{p}{n-j-1}} & =\left[\frac{1}{n} \int_{S^{n-1}} \rho_{K \widetilde{+}_{p} L}(u)^{n-j-1} \rho_{Q}(u) d u\right]^{\frac{p}{n-j-1}}=\left[\frac{1}{n} \int_{S^{n-1}}\left(\rho_{K \widetilde{+}_{p} L}(u)^{p}\right)^{\frac{n-j-1}{p}} \rho_{Q}(u) d u\right]^{\frac{p}{n-j-1}} \\
& =\left[\frac{1}{n} \int_{S^{n-1}}\left(\rho_{K}(u)^{p}+\rho_{L}(u)^{p}\right)^{\frac{n-j-1}{p}} \rho_{Q}(u) d u\right]^{\frac{p}{n-j-1}} \geq\left[\frac{1}{n} \int_{S^{n-1}} \rho_{K}(u)^{n-j-1} \rho_{Q}(u) d u\right]^{\frac{p}{n-j-1}} \\
& +\left[\frac{1}{n} \int_{S^{n-1}} \rho_{L}(u)^{n-j-1} \rho_{Q}(u) d u\right]^{\frac{p}{n-j-1}}=\widetilde{W}_{j}(K, Q)^{\frac{p}{n-j-1}}+\widetilde{W}_{j}(L, Q)^{\frac{p}{n-j-1}}
\end{aligned}
$$

This yields inequality (15).

According to the equality condition of Minkowski integral inequality, we easily see equality holds in (15) if and only if $K$ and $L$ are dilates.

Similarly, if $0<p<n-1$ and $0 \leq j<n-p-1$, then $\frac{n-j-1}{p}>1$. Hence, by the Minkowski integral inequality, we know that inequality (15) is reverse, i.e., inequality (16) and its equality condition are obtained.

Lemma 3.4 [10] If $K, L \in \mathcal{S}_{o}^{n}$ and $i, j=0,1, \cdots, n-2$, then

$$
\widetilde{W}_{i}\left(K, \Psi_{j} L\right)=\widetilde{W}_{j}\left(L, \Psi_{i} K\right) .
$$


Proof of Theorem 1.2 For $i=0,1, \cdots, n-2$, if $p<0$ and $0 \leq j<n-1$, then by (15) and (17) we have for any $M \in \mathcal{S}_{o}^{n}$,

$$
\begin{aligned}
\widetilde{W}_{i}(M, & \left.\Psi_{j}\left(K \widetilde{+}_{p} L\right)\right)^{\frac{p}{n-j-1}}=\widetilde{W}_{j}\left(K \widetilde{+}_{p} L, \Psi_{i} M\right)^{\frac{p}{n-j-1}} \\
& \geq \widetilde{W}_{j}\left(K, \Psi_{i} M\right)^{\frac{p}{n-j-1}}+\widetilde{W}_{j}\left(L, \Psi_{i} M\right)^{\frac{p}{n-j-1}} \\
& =\widetilde{W}_{i}\left(M, \Psi_{j} K\right)^{\frac{p}{n-j-1}}+\widetilde{W}_{i}\left(M, \Psi_{j} L\right)^{\frac{p}{n-j-1}} .
\end{aligned}
$$

This together with Minkowski inequality (11), and notice that $\frac{p}{n-j-1}<0$, we get

$$
\begin{aligned}
& \widetilde{W}_{i}\left(M, \Psi_{j}\left(K \widetilde{+}_{p} L\right)\right)^{\frac{p}{n-j-1}} \geq \widetilde{W}_{i}\left(M, \Psi_{j} K\right)^{\frac{p}{n-j-1}}+\widetilde{W}_{i}\left(M, \Psi_{j} L\right)^{\frac{p}{n-j-1}} \\
& \geq \widetilde{W}_{i}(M)^{\frac{p(n-i-1)}{(n-i)(n-j-1)}}\left[\widetilde{W}_{i}\left(\Psi_{j} K\right)^{\frac{p}{(n-i)(n-j-1)}}+\widetilde{W}_{i}\left(\Psi_{j} L\right)^{\frac{p}{(n-i)(n-j-1)}}\right] .
\end{aligned}
$$

Let $M=\Psi_{j}\left(K \widetilde{+}_{p} L\right)$ in above inequality, we obtain inequality (4).

By the equality conditions of inequalities (11) and (15), we see that equality holds in (4) if and only if $K$ and $L$ are dilates.

In the same method, if $0<p<n-1$ and $0 \leq j<n-p-1$, then inequality (5) and its equality condition can be obtained.

\section{Acknowledgements}

The author wants to express earnest thankfulness for the referees who provided extremely precious and helpful comments and suggestions. Research is supported in part by the Natural Science Foundation of China (Grant No.11371224).

\section{References}

[1] R. J. Gardner, "Geometric Tomography", Second ed., Cambridge Univ. Press, Cambridge, 2006.

[2] E. Lutwak, "intersection bodies and dual mixed volumes", Adv. Math., 71 (1988), 531-538.

[3] R. J. Gardner, "Intersection bodies and the BusemannPetty problem", Trans. Amer. Math. Soc., 342 (1994) 435-445.

[4] A. Koldlbsky, "A functional analytic approach to intersection bodies", Geom. Funct. Anal., 10 (2000), 1507-1526.

[5] G. S. Leng and C. J. Zhao, "Inequalities for dual quermassintegrals of mixed intersction bodies", Proc. Indian Acad. Sci., 115 (2003), 79-91.

[6] G. S. Leng and C. J. Zhao, "Brunn-Minkowski inequality for mixed intersection bodies", J. Math. Anal. Appl., 301 (2005), 115-123.

[7] R. Schneider, "Convex Bodies: The Brunn-Minkowski theory", 2nd edn, Cambridge University Press, Cambridge, 2014.

[8] G. Y. Zhang, "Centered bodies and dual mixed volumes", Trans. Amer. Math. Soc., 345 (1994), 777-801.
[9] G. Y. Zhang, "A positive solution to the Busemann-Petty problem in $R^{4}$ ", Ann. of Math., 149 (1999), 2: 535-543.

[10] F. E. Schuster, "Volume inequalities and additive maps of convex bodies", Mathematika, 53 (2006), 211-234.

[11] F. E. Schuster, "Valuations and Busemann-Petty type problems", Adv. Math., 219 (2008), 344-368.

[12] W. Wang, L. J. Liu and B. W. He " $L_{p}$ radial BlaschkeMinkowski homomorphisms", Taiwan J. Math., 15 (2011), 1183-1199.

[13] B. Chen and W. D. Wang, "A type of Busemann-Petty problems for Blaschke-Minkowski homomorphisms", Wuhan University Journal of Natural Sciences, 23 (2018), 289-294.

[14] B. Chen and W. D. Wang, "Some inequalities for $L_{p}$ radial Blaschke-Minkowski homomorphisms", Quaest. Math., 42 (2019), 391-405.

[15] Y. B. Feng, W. D. Wang and J. Yuan, "Differences of quermass- and dual quermassintegrals of Blaschke-Minkowski and radial Blaschke-Minkowski homomorphisms", B. Belg. Math. Soc-Sim, 21 (2014), 577-592.

[16] L. J. Liu, "Mixed radial Blaschke-Minkowski homomorphisms and comparison of volumes", Math. Inequal. Appl., 16 (2013), 401-412.

[17] Z. H. Shen and W. D. Wang, " $L_{p}$ radial BlaschkeMinkowski homomorphisms and $L_{p}$ dual affine surface areas", Mathematics, 7 (2019), 343: 14 pages.

[18] W. D. Wang, H. P. Chen and Y. Y. Zhang, "BusemannPetty problem for the $i$-th radial Blaschke-Minkowski homomorphisms", Filomat, 32 (2018), 6819-6827.

[19] B. Wei, W. D. Wang and F. H. Lu, "Inequalities for radial Blaschke-Minkowski homomorphisms", Ann. Pol. Math., 113 (2015), 243-253.

[20] C. J. Zhao, "Radial Blaschke-Minkowski homomorphisms and volume differences", Geom. Dedicata, 154 (2011), 81-91. 
[21] C. J. Zhao, "On radial Blaschke-Minkowski homomorphisms”, Geom. Dedicata, 167 (2013), 1-10.

[22] C. J. Zhao, “On raidal and polar Blaschke-Minkowski homomorphisms", Proc. Amer. Math. Soc., 141 (2013), 667-676.

[23] C. J. Zhao, “On Blaschke-Minkowski homomorphisms and radial Blaschke-Minkowski homomorphisms", J. Geom. Anal., 25 (2015), 1-16.

[24] X. Zhao and W. D. Wang, "Brunn-Minkowski inequalities for the $L_{p}$ and $L_{p}$ radial Blaschke-Minkowski homomorphisms", IAENG International Journal of Applied Mathematics, 49 (2019), 1-5.

[25] Y. Zhou and W. D. Wang, "Some Brunn-Minkowski type inequalities for $L_{p}$ radial Blaschke-Minkowski homomorphisms", J. Inequal. Appl., 2016 (2016), 183, 11 pages.

[26] H. Busemann, "Volum in terms of concurrent crosssections", Pacofoc J. Math., 3 (1953), 1-12.

[27] R. J. Gardner, “The Brunn-Minkowski inequality”, Bull. Amer. Math. Soc., 39 (2002), 355-405.

[28] G. Y. Zhang, "Convolutions, transforms, and convex bodies”, Proc. London Math. Soc., 78 (1999), 77-115.

[29] J. Y. Wang and W. D. Wang, "General $L_{p}$-dual Blaschke bodies and the applications", J. Inequal. Appl., 2015 (2015), 233, 11 pages.

[30] E. Lutwak, "Dual mixed volumes", Pacific J. Math., 58 (1975), 531-538. 